INPLASY

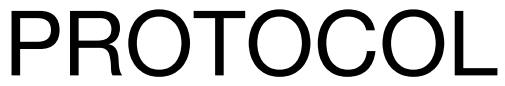

To cite: Gui et al. Chinese herbal injections for radiation pneumonitis: A protocol for systematic review and metaanalyses. Inplasy protocol 202210106. doi:

10.37766/inplasy2022.1.0106

Received: 20 January 2022

Published: 20 January 2022

Corresponding author:

Yuerong Gui

guiyuerong@163.com

Author Affiliation:

Guang'anmen Hospital, China

Academy of Chinese Medical

Sciences.

Support: 82074239.

Review Stage at time of this submission: The review has not yet started.

Conflicts of interest:

None declared.

\section{Chinese herbal injections for radiation pneumonitis: A protocol for systematic review and meta-analyses}

Gui, Y1; Pang, Q2; Wang, S3; Dong, J4; Wang, D5; Ma, X6; Wang, $X^{7} ; \mathrm{Hu}, \mathrm{S}^{8} ; \mathrm{Hou}, \mathrm{W}^{9}$.

Review question / Objective: Radiation pneumonitis is a common dose-limiting factor in radiotherapy for thoracic malignancies, and its treatment encounters a bottleneck. As an important adjuvant treatment method, Chinese herbal injections(CHIs) have been used in the treatment of radiation pneumonitis(RP), and clinical studies have shown certain therapeutic advantages. However, the efficacy and safety of all type of CHIs for RP have not been evaluated comprehensively.

Information sources: The Cochrane Library, PubMed, EMBASE, SinoMed, CNKI, VIP, and Wan Fang Databases were systematically searched from inceptions until January 2022. References about related systematic reviews in this research field were searched manually and relevant experts in the field were consulted.

INPLASY registration number: This protocol was registered with the International Platform of Registered Systematic Review and Meta-Analysis Protocols (INPLASY) on 20 January 2022 and was last updated on 20 January 2022 (registration number INPLASY202210106).

\section{INTRODUCTION}

Review question / Objective: Radiation pneumonitis is a common dose-limiting factor in radiotherapy for thoracic malignancies, and its treatment encounters a bottleneck. As an important adjuvant treatment method, Chinese herbal injections(CHIs) have been used in the treatment of radiation pneumonitis(RP), and clinical studies have shown certain therapeutic advantages. However, the efficacy and safety of all type of CHIs for 
R Pave not been evaluated comprehensively.

Condition being studied: Radiation pneumonitis.

\section{METHODS}

Participant or population: Patients (aged 18 years or older) with RP, which should be confirmed according to the clinical diagnostic standard.[2, 16] According to the severity of clinical symptoms, the patients were divided into grades 0-5 according to the Radiation Therapy Oncology Group (RTOG) standard, radiation pneumonitis $\geq$ grade 2 , and patients requiring active treatment, the course of disease was less than 6 months. There are no limitations in gender, education, race, or nationality.

Intervention: On the basis of the control group, use CHls intravenously. There is no limitation on the dose and intervention time of CHIs.

Comparator: Placebo/no intervention, or conventional treatment such as Glucocorticoid and/or antibiotics.

Study designs to be included: Randomized controlled trials.

Eligibility criteria: Type of study. Randomized controlled trials (RCTs) that investigated the effect of $\mathrm{CHI}$ for the treatment of RP will be included. No language limitation exists.2.2.2. Participants. Patients (aged 18 years or older) with RP, which should be confirmed according to the clinical diagnostic standard. According to the severity of clinical symptoms, the patients were divided into grades $0-5$ according to the Radiation Therapy Oncology Group (RTOG) standard, radiation pneumonitis $\geq$ grade 2 , and patients requiring active treatment, the course of disease was less than 6 months. There are no limitations in gender, education, race, or nationality.2.2.3. Interventions. Control group: placebo/no intervention, or conventional treatment such as Glucocorticoid and/or antibiotics;
Test group: On the basis of the control group, use CHls intravenously. There is no limitation on the dose and intervention time of CHIs.In addition to the above treatment, both groups can receive the same basic treatment, such as oxygen absorption, cough clearance, phlegm reduction, and bronchiectasis. For specific treatment methods, refer to the Expert consensus for the Diagnosis and treatment of radiation induced lung injury.

Information sources: The Cochrane Library, PubMed, EMBASE, SinoMed, CNKI, VIP, and Wan Fang Databases were systematically searched from inceptions until January 2022. References about related systematic reviews in this research field were searched manually and relevant experts in the field were consulted.

Main outcome(s): Total effective rate: According to UICC criterias for judging the efficacy of RP, [17]the pneumonia remissions were evaluated as complete remission (CR), partial remission (PR), and not cured (NC).

Additional outcome(s): 1. Quality of Life (Qol). Patient's Qol was measured before and after treatment using the Karnofsky performance status (KPS) scale. An increase of 10 points or more on the KPS score was considered improvement. 2. Clinical Symptoms and Signs. Duration of fever, cough, asthma exacerbation, and colored sputum was evaluated. 3. Inflammatory Cytokines. Interleukin-6 (IL-6), TGF- $\beta$, and TNF- $\alpha$ cytokines in plasma were evaluated. 4. Incidence of Adverse Events. Adverse events related to CHls intervention were extracted.

Quality assessment / Risk of bias analysis: Two reviewers will assess the risk of bias of the included studies by "Risk of Bias Assessment Tool" of the Cochrane Handbook for RCTs. The evaluation contents include random sequence generation, allocation concealment, blinding of participants and personnel, blinding of outcome assessment, incomplete outcome data, selective reporting, and other biases. Each item is 
divided into "high risk", "unclear risk", and "low risk". Any inconsistencies will be determined in consultation with the third reviewer.

Strategy of data synthesis: Data Synthesis. Statistical analysis will be performed with Review Manager software 5.3. Relative risk (RR) is used to evaluate the effect size for binary variables, and the mean difference (MD) is used as the efficacy analysis statistic for continuous variables. Heterogeneity between results will be assessed by the value of $P$ and $12, P>0.1$ and $12<50 \%$ indicate small heterogeneity; $P<0.1$ and $I 2 \geq 50 \%$ indicate high heterogeneity. The fixed-effect model was used in homogeneity data merging and the random-effects model was suitable for the merging of heterogeneous data.If there is clinical and methodological heterogeneity, subgroup analyses, meta-regression analyses, or descriptive analyses were performed as appropriate.

Subgroup analysis: If the results to be analyzed are heterogeneous, analyze the source of the heterogeneity, and perform subgroup analysis according to the patient's age, RTOG classification, the type of CHIs, the duration of treatment, or medication dosage, etc.

Sensitivity analysis: If the risk of bias of the studies is high, sensitivity analysis will be performed to investigate the asymmetry of funnel plots to exclude low-quality studies. Evaluate whether the results of the metaanalysis are stable and reliable.

Country(ies) involved: China.

Keywords: radiation therapy, Chinese herbal injections, efficacy, randomized controlled trial, systematic review.

Contributions of each author:

Author 1 - Yuerong Gui.

Author 2 - Qing Pang.

Author 3 - Shuo Wang.

Author 4 - Jun Dong.

Author 5 - Dandan Wang.

Author 6 - Xiumei Ma.

Author 7 - Xinyan Wang.
Author 8 - Shuanghang Hu.

Author 9 - Wei Hou. 